\title{
No place for particles in relativistic quantum theories?
}

\author{
Hans Halvorson and Rob Clifton \\ Department of Philosophy, University of Pittsburgh \\ hphst1@pitt.edu,rclifton@pitt.edu
}

\begin{abstract}
Several recent arguments purport to show that there can be no relativistic, quantum-mechanical theory of localizable particles and, thus, that relativity and quantum mechanics can be reconciled only in the context of quantum field theory. We point out some loopholes in the existing arguments, and we provide two no-go theorems to close these loopholes. However, even with these loopholes closed, it does not yet follow that relativity plus quantum mechanics exclusively requires a field ontology, since relativistic quantum field theory itself might permit an ontology of localizable particles supervenient on the fundamental fields. Thus, we provide another no-go theorem to rule out this possibility. Finally, we allay potential worries about this conclusion by arguing that relativistic quantum field theory can nevertheless explain the possibility of "particle detections", as well as the pragmatic utility of "particle talk."
\end{abstract}

\section{Introduction}

It is a widespread belief, at least within the physics community, that there is no particle mechanics that is simultaneously relativistic and quantum-theoretic; and, thus, that the only relativistic quantum theory is a field theory. This belief has received much support in recent years in the form of rigorous "no-go theorems" by Malament (1996) and Hegerfeldt (1998a, 1998b). In particular, Hegerfeldt shows that in a generic quantum theory (relativistic or non-relativistic), if there are states with localized particles, and if there is a lower bound on the system's energy, then superluminal spreading of the wavefunction must occur. Similarly, Malament shows the inconsistency of a few intuitive desiderata for a relativistic, quantum-mechanical theory of (localizable) particles. Thus, it appears that there is a fundamental conflict between the demands of relativistic causality and the requirements of a theory of localizable particles. 
What is the philosophical lesson of this apparent conflict between relativistic causality and localizability? One the one hand, if we believe that the assumptions of Malament's theorem must hold for any theory that is descriptive of our world, then it follows that our world cannot be correctly described by a particle theory. On the other hand, if we believe that our world can be correctly described by a particle theory, then one (or more) of the Malament's assumptions must be false. Malament clearly endorses the first response; that is, he argues that his theorem entails that there is no relativistic quantum mechanics of localizable particles (insofar as any relativistic theory precludes act-outcome correlations at spacelike separation). Others, however, have argued that the assumptions of Malament's theorem need not hold for any relativistic, quantum-mechanical theory (cf. Fleming and Butterfield 1999), or that we cannot judge the truth of the assumptions until we resolve the interpretive issues of elementary quantum mechanics (cf. Barrett 2000).

Although we do not think that these arguments against Malament's assumptions succeed, there are other reasons to doubt that Malament's theorem is sufficient to support a sound argument against the possibility of a relativistic quantum mechanics of localizable particles. First, Malament's theorem depends on a specific assumption about the structure of Minkowski spacetime - a "no preferred reference frame" assumption - that could be seen as having less than full empirical warrant. Second, Malament's theorem establishes only that there is no relativistic quantum mechanics in which particles can be completely localized in spatial regions with sharp boundaries; it leaves open the possibility that there might be a relativistic quantum mechanics of "unsharply" localized particles. In this paper, we present two new no-go theorems which, together, suffice to close these loopholes in the argument against relativistic quantum mechanics. First, we present a strengthened no-go theorem that subsumes the results of Malament and Hegerfeldt, and which does not depend on the "no preferred frame" assumption (Theorem 11). Second, we derive a generalized version of Malament's theorem that shows that there is no relativistic quantum mechanics of "unsharply" localized particles (Theorem 2).

However, it would be a mistake to think that these result show-or, are intended to show - that a field ontology, rather than a particle ontology, is appropriate for relativistic quantum theories. While these results show that there are no position observables that satisfy certain relativistic constraints, quantum field theories-both relativistic and non-relativistic-already reject the notion of position observables in favor of "localized" field observables. Thus, no-go results against relativistic position operators have nothing to say about the possibility that relativistic quantum field theory might permit a "particle interpretation," in which localized particles are supervenient on the underlying localized field observables. To exclude this latter possibility, we formulate (in Section 6) a necessary condition for a generic quantum theory to permit a particle interpretation, and we then show 
that this condition fails in any relativistic theory (Theorem 3).

Since our world is presumably both relativistic and quantum-theoretic, these results show that there are no localizable particles. However, in Section 7 we shall argue that relativistic quantum field theory itself warrants an approximate use of "particle talk" that is sufficient to save the phenomena.

\section{Malament's Theorem}

Malament's theorem shows the inconsistency of a few intuitive desiderata for a relativistic quantum mechanics of (localizable) particles. It strengthens previous results (e.g., Schlieder 1971) by showing that the assumption of "no superluminal wavepacket spreading" can be replaced by the weaker assumption of "microcausality," and by making it clear that Lorentz invariance is not needed to derive a conflict between relativistic causality and localizability.

In order to present Malament's result, we assume that our background spacetime $M$ is an affine space, with a foliation $\mathcal{S}$ into spatial hyperplanes. (For ease, we can think of an affine space as a vector space, so long as we do not assign any physical significance to the origin.) This will permit us to consider a wide range of relativistic (e.g., Minkowski) as well as non-relativistic (e.g., Galilean) spacetimes. The pure states of our quantum-mechanical system are given by rays in some Hilbert space $\mathcal{H}$. We assume that there is a mapping $\Delta \mapsto E_{\Delta}$ of bounded subsets of hyperplanes in $M$ into projections on $\mathcal{H}$. We think of $E_{\Delta}$ as representing the proposition that the particle is localized in $\Delta$; or, from a more operational

point of view, $E_{\Delta}$ represents the proposition that a position measurement is certain to find the particle within $\Delta$. We also assume that there is a strongly continuous representation $\mathbf{a} \mapsto U(\mathbf{a})$ of the translation group of $M$ in the unitary operators on $\mathcal{H}$. Here strong continuity means that for any unit vector $\psi \in \mathcal{H},\langle\psi, U(\mathbf{a}) \psi\rangle \rightarrow 1$ as $\mathbf{a} \rightarrow 0$; and it is equivalent (via Stone's theorem) to the assumption that there are energy and momentum observables for the particle. If all of the preceding conditions hold, we say that the triple $\left(\mathcal{H}, \Delta \mapsto E_{\Delta}, \mathbf{a} \mapsto U(\mathbf{a})\right)$ is a localization system over $M$.

The following conditions should hold for any localization system-either relativistic or non-relativistic - that describes a single particle.

Localizability: If $\Delta$ and $\Delta^{\prime}$ are disjoint subsets of a single hyperplane, then $E_{\Delta} E_{\Delta^{\prime}}=0$.

Translation covariance: For any $\Delta$ and for any translation a of $M$, $U(\mathbf{a}) E_{\Delta} U(\mathbf{a})^{*}=E_{\Delta+\mathbf{a}}$. 
Energy bounded below: For any timelike translation a of $M$, the generator $H(\mathbf{a})$ of the one-parameter group $\{U(t \mathbf{a}): t \in \mathbb{R}\}$ has a spectrum bounded from below.

We recall briefly the motivation for each of these conditions. "Localizability" says that the particle cannot be detected in two disjoint spatial sets at a given time. "Translation covariance" gives us a connection between the symmetries of the spacetime $M$ and the symmetries of the quantum-mechanical system. In particular, if we displace the particle by a spatial translation a, then the original wavefunction $\psi$ will transform to some wavefunction $\psi_{\mathbf{a}}$. Since the statistics for the displaced detection experiment should be identical to the original statistics, we have $\left\langle\psi, E_{\Delta} \psi\right\rangle=\left\langle\psi_{\mathbf{a}}, E_{\Delta+\mathbf{a}} \psi_{\mathbf{a}}\right\rangle$. By Wigner's theorem, however, the symmetry is implemented by some unitary operator $U(\mathbf{a})$. Thus, $U(\mathbf{a}) \psi=\psi_{\mathbf{a}}$, and $U(\mathbf{a}) E_{\Delta} U(\mathbf{a})^{*}=E_{\Delta+\mathbf{a}}$. In the case of time translations, the covariance condition entails that the particle has unitary dynamics. (This might seem to beg the question against a collapse interpretation of quantum mechanics; we dispell this worry at the end of this section.) Finally, the "energy bounded below" condition asserts that, relative to any free-falling observer, the particle has a lowest possible energy state. If it were to fail, we could extract an arbitrarily large amount of energy from the particle as it drops down through lower and lower states of energy.

We now turn to the "specifically relativistic" assumptions needed for Malament's theorem. The special theory of relativity entails that there is a finite upper bound on the speed at which (detectable) physical disturbances can propagate through space. Thus, if $\Delta$ and $\Delta^{\prime}$ are distant regions of space, then there is a positive lower bound on the amount of time it should take for a particle localized in $\Delta$ to travel to $\Delta^{\prime}$. We can formulate this requirement precisely by saying that for any timelike translation a, there is an $\epsilon>0$ such that, for every state $\psi$, if $\left\langle\psi, E_{\Delta} \psi\right\rangle=1$ then $\left\langle\psi, E_{\Delta^{\prime}+t \mathbf{a}} \psi\right\rangle=0$ whenever $0 \leq t<\epsilon$. This is equivalent to the following assumption.

Strong causality: If $\Delta$ and $\Delta^{\prime}$ are disjoint subsets of a single hyperplane, and if the distance between $\Delta$ and $\Delta^{\prime}$ is nonzero, then for any timelike translation a, there is an $\epsilon>0$ such that $E_{\Delta} E_{\Delta^{\prime}+t a}=0$ whenever $0 \leq t<\epsilon$.

(Note that strong causality entails localizability.) Although strong causality is a reasonable condition for relativistic theories, Malament's theorem requires only the following weaker assumption (which he himself calls "locality").

Microcausality: If $\Delta$ and $\Delta^{\prime}$ are disjoint subsets of a single hyperplane, and if the distance between $\Delta$ and $\Delta^{\prime}$ is nonzero, then for any timelike translation a, there is an $\epsilon>0$ such that $\left[E_{\Delta}, E_{\Delta^{\prime}+t \mathbf{a}}\right]=0$ whenever $0 \leq t<\epsilon$. 
If $E_{\Delta}$ can be measured within $\Delta$, microcausality is equivalent to the assumption that a measurement within $\Delta$ cannot influence the statistics of measurements performed in regions that are spacelike to $\Delta$ (see Malament 1996, 5). Conversely, a failure of microcausality would entail the possibility of act-outcome correlations at spacelike separation. Note that both strong and weak causality make sense for nonrelativistic spacetimes (as well as for relativistic spacetimes); though, of course, we should not expect either causality condition to hold in the non-relativistic case.

Theorem (Malament). Let $\left(\mathcal{H}, \Delta \mapsto E_{\Delta}, \mathbf{a} \mapsto U(\mathbf{a})\right)$ be a localization system over Minkowski spacetime that satisfies:

\section{Localizability}

\section{Translation covariance}

\section{Energy bounded below}

\section{Microcausality}

Then $E_{\Delta}=0$ for all $\Delta$.

Thus, in every state, there is no chance that the particle will be detected in any local region of space. As Malament claims, this serves as a reductio ad absurdum of any relativistic quantum mechanics of a single (localizable) particle.

Several authors have claimed that Malament's theorem is not sufficient to rule out a relativistic quantum mechanics of localizable particles. In particular, these authors argue that it is not reasonable to expect the conditions of Malament's theorem to hold for any relativistic, quantum-mechanical theory of particles. For example, Dickson (1997) argues that a 'quantum' theory does not need a position operator (equivalently, a system of localizing projections) in order to treat position as a physical quantity; Barrett (2000) argues that time-translation covariance is suspect; and Fleming and Butterfield (1999) argue that the microcausality assumption is not warranted by special relativity. We now show, however, that none of these arguments is decisive against the assumptions of Malament's theorem.

Dickson $(1997,214)$ cites the Bohmian interpretation of the Dirac equation as a counterexample to the claim that any 'quantum' theory must represent position by an operator. In order to see what Dickson might mean by this, recall that the Dirac equation admits both positive and negative energy solutions. If $\mathcal{H}$ denotes the Hilbert space of all (both positive and negative energy) solutions, then we may define the 'standard position operator' $Q$ by setting $Q \psi(\boldsymbol{x})=\boldsymbol{x} \cdot \psi(\boldsymbol{x})$ (Thaller 1992, 7). If, however, we restrict to the Hilbert space $\mathcal{H}_{\text {pos }} \subset \mathcal{H}$ of positive energy solutions, then the probability density given by the Dirac wavefunction does 
not correspond to a self-adjoint position operator (Thaller 1992, 32). According to Holland $(1993,502)$, this lack of a position operator on $\mathcal{H}_{\text {pos }}$ precludes a Bohmian interpretation of $\psi(\boldsymbol{x})$ as a probability amplitude for finding the particle in an elementary volume $d^{3} \boldsymbol{x}$ around $\boldsymbol{x}$.

Since the Bohmian interpretation of the Dirac equation uses all states (both positive and negative energy), and the corresponding position observable $Q$, it is not clear what Dickson means by saying that the Bohmian interpretation of the Dirac equation dispenses with a position observable. Moreover, since the energy is not bounded below in $\mathcal{H}$, this would not in any case give us a counterexample to Malament's theorem. However, Dickson could have developed his argument by appealing to the positive energy subspace $\mathcal{H}_{\text {pos }}$. In this case, we can talk about positions despite the fact that we do not have a position observable in the usual sense. In particular, we shall show in Section 5 that, for talk about positions, it suffices to have a family of "unsharp" localization observables. (And, yet, we shall show that relativistic quantum theories do not permit even this attenuated notion of localization.)

Barrett (2000) argues that the significance of Malament's theorem cannot be assessed until we have solved the measurement problem:

If we might have to violate the apparently weak and obvious assumptions that go into proving Malament's theorem in order to get a satisfactory solution to the measurement problem, then all bets are off concerning the applicability of the theorem to the detectible entities that inhabit our world. (Barrett 2000, 16)

In particular, a solution to the measurement problem may require that we abandon unitary dynamics. But if we abandon unitary dynamics, then the translation covariance condition does not hold, and we need not accept the conclusion that there is no relativistic quantum mechanics of (localizable) particles.

Unfortunately, it is not clear that we could avoid the upshot of Malament's theorem by moving to a collapse theory. Existing (non-relativistic) collapse theories take the empirical predictions of quantum theory seriously. That is, the "statistical algorithm" of quantum mechanics is assumed to be at least approximately correct; and collapse is introduced only to ensure that we obtain determinate properties at the end of a measurement. However, in the present case, Malament's theorem shows that the statistical algorithm of any quantum theory predicts that if there are local particle detections, then act-outcome correlations are possible at spacelike separation. Thus, if a collapse theory is to stay close to these predictions, it too would face a conflict between localizability and relativistic causality.

Perhaps, then, Barrett is suggesting that the price of accomodating localizable particles might be a complete abandonment of unitary dynamics, even at the level 
of a single particle. In other words, we may be forced to adopt a collapse theory without having any underlying (unitary) quantum theory. But even if this is correct, it wouldn't count against Malament's theorem, which was intended to show that there is no relativistic quantum theory of localizable particles. Furthermore, noting that Malament's theorem requires unitary dynamics is one thing; it would be quite another thing to provide a model in which there are localizable particles-at the price of non-unitary dynamics - but which is also capable of reproducing the wellconfirmed quantum interference effects at the micro-level. Until we have such a model, pinning our hopes for localizable particles on a failure of unitary dynamics is little more than wishful thinking.

Like Barrett, Fleming (Fleming and Butterfield 1999, 158ff) disagrees with the reasonableness of Malament's assumptions. Unlike Barrett, however, Fleming provides a concrete model in which there are localizable particles (viz., using the Newton-Wigner position operator as a localizing observable) and in which Malament's microcausality assumption fails. Nonetheless, Fleming argues that this failure of microcausality is perfectly consistent with relativistic causality.

According to Fleming, the property "localized in $\Delta$ " (represented by $E_{\Delta}$ ) need not be detectable within $\Delta$. As a result, $\left[E_{\Delta}, E_{\Delta^{\prime}}\right] \neq 0$ does not entail that it is possible to send a signal from $\Delta$ to $\Delta^{\prime}$. However, by claiming that local beables need not be local observables, Fleming undercuts the primary utility of the notion of localization, which is to indicate those physical quantities that are operationally accessible in a given region of spacetime. Indeed, it is not clear what motivation there could be-aside from indicating what is locally measurable-for assigning observables to spatial regions. If $E_{\Delta}$ is not measurable in $\Delta$, then why should we say that " $E_{\Delta}$ is localized in $\Delta^{\prime}$ "? Why not say instead that " $E_{\Delta}$ is localized in $\Delta^{\prime \prime}$ " (where $\Delta^{\prime} \neq \Delta$ )? Does either statement have any empirical consequences and, if so, how do their empirical consequences differ? Until these questions are answered, we maintain that local beables are always local observables; and a failure of microcausality would entail the possibility of act-outcome correlations at spacelike separation. Therefore, the microcausality assumption is an essential feature of any relativistic quantum theory with "localized" observables. (For a more detailed argument along these lines, see Halvorson 2001, Section 6.)

Thus, the arguments against the four (explicit) assumptions of Malament's theorem are unsuccessful; these assumptions are perfectly reasonable, and we should expect them to hold for any relativistic, quantum-mechanical theory. However, there is another difficulty with the argument against any relativistic quantum mechanics of (localizable) particles: Malament's theorem makes tacit use of specific features of Minkowski spacetime which—some might claim-have less than perfect empirical support. First, the following example shows that Malament's theorem fails if there is a preferred reference frame. 
Example 1. Let $M=\mathbb{R}^{1} \oplus \mathbb{R}^{3}$ be full Newtonian spacetime (with a distinguished timelike direction a). To any set of the form $\{(t, x): x \in \Delta\}$, with $t \in \mathbb{R}$, and $\Delta$ a bounded open subset of $\mathbb{R}^{3}$, we assign the spectral projection $E_{\Delta}$ of the position operator for a particle in three dimensions. Let $H(\mathbf{a})=0$ so that $U(t \mathbf{a})=e^{i t 0}=I$ for all $t \in \mathbb{R}$. Since the energy in every state is zero, the energy condition is trivially satisfied.

Note, however, that if the background spacetime is not regarded as having a distinguished timelike direction, then this example violates the energy condition. Indeed, the generator of an arbitrary timelike translation has the form

$$
H(\mathbf{b})=\mathbf{b} \cdot \mathbf{P}=b_{0} 0+b_{1} P_{1}+b_{2} P_{2}+b_{3} P_{3}=b_{1} P_{1}+b_{2} P_{2}+b_{3} P_{3},
$$

where $\mathbf{b}=\left(b_{0}, b_{1}, b_{2}, b_{3}\right) \in \mathbb{R}^{4}$ is a timelike vector, and $P_{i}$ are the three orthogonal components of the total momentum. But since each $P_{i}$ has spectrum $\mathbb{R}$, the spectrum of $H(\mathbf{b})$ is not bounded from below when $\mathbf{b} \neq \mathbf{a}$.

Malament's theorem does not require the full structure of Minkowski spacetime (e.g., the Lorentz group). Rather, it suffices to assume that the affine space $M$ satisfies the following condition.

No absolute velocity: Let a be a spacelike translation of $M$. Then there is a pair $(\mathbf{b}, \mathbf{c})$ of timelike translations of $M$ such that $\mathbf{a}=\mathbf{b}-\mathbf{c}$.

Despite the fact that "no absolute velocity" is a feature of all post-Galilean spacetimes, there are some who claim that the existence of a (undetectable) preferred reference frame is perfectly consistent with the empirical evidence on which relativistic theories are based (cf. Bell 1987, Chap. 9). What is more, the existence of a preferred frame is an absolutely essential feature of a number of "realistic" interpretations of quantum theory (cf. Maudlin 1994, Chap. 7). Thus, this tacit assumption of Malament's theorem has the potential to be a major source of contention for those wishing to maintain that there can be a relativistic quantum mechanics of localizable particles.

There is a further worry about the generality of Malament's theorem: It is not clear whether the result can be expected to hold for arbitrary relativistic spacetimes, or whether it is an artifact of peculiar features of Minkowski spacetime (e.g., that space is infinite). To see this, suppose that $M$ is an arbitrary globally hyperbolic manifold. (That is, $M$ is a manifold that permits at least one foliation $\mathcal{S}$ into spacelike hypersurfaces). Although $M$ will not typically have a translation group, we suppose that $M$ has a transitive Lie group $G$ of diffeomorphisms. (Just as a manifold is locally isomorphic to $\mathbb{R}^{n}$, a Lie group is locally isomorphic to a group of translations.) We require that $G$ has a representation $g \mapsto U(g)$ in the 
unitary operators on $\mathcal{H}$; and, the translation covariance condition now says that $E_{g(\Delta)}=U(g) E_{\Delta} U(g)^{*}$ for all $g \in G$.

The following example shows that Malament's theorem fails even for the very simple case where $M$ is a two-dimensional cylinder.

Example 2. Let $M=\mathbb{R} \oplus S^{1}$, where $S^{1}$ is the one-dimensional unit circle, and let $G$ denote the Lie group of timelike translations and rotations of $M$. It is not difficult to construct a unitary representation of $G$ that satisfies the energy bounded below condition. (We can use the Hilbert space of square-integrable functions from $S^{1}$ into $\mathbb{C}$, and the procedure for constructing the unitary representation is directly analogous to the case of a single particle moving on a line.) Fix a spacelike hypersurface $\Sigma$, and let $\mu$ denote the normalized rotation-invariant measure on $\Sigma$. For each open subset $\Delta$ of $\Sigma$, let $E_{\Delta}=I$ if $\mu(\Delta) \geq 2 / 3$, and let $E_{\Delta}=0$ if $\mu(\Delta)<2 / 3$. Then localizability holds, since for any pair $\left(\Delta, \Delta^{\prime}\right)$ of disjoint open subsets of $\Sigma$, either $\mu(\Delta)<2 / 3$ or $\mu\left(\Delta^{\prime}\right)<2 / 3$.

Nonetheless, Examples 1 and 2 hardly serve as physically interesting counterexamples to a strengthened version of Malament's theorem. In particular, in Example 11 the energy is identically zero, and therefore the probability for finding the particle in a given region of space remains constant over time. In Example 2, the particle is localized in every region of space with volume greater than $2 / 3$, and the particle is never localized in a region of space with volume less than $2 / 3$. In the following two sections, then, we will formulate explicit conditions to rule out such pathologies, and we will use these conditions to derive a strengthened version of Malament's theorem that applies to generic spacetimes.

\section{Hegerfeldt's Theorem}

Hegerfeldt's (1998a, 1998b) recent results on localization apply to arbitrary (globally hyperbolic) spacetimes, and they do not make us of the "no absolute velocity" condition. Thus, we will suppose henceforth that $M$ is a globally hyperbolic spacetime, and we will fix a foliation $\mathcal{S}$ of $M$, as well as a unique isomorphism between any two hypersurfaces in this foliation. If $\Sigma \in \mathcal{S}$, we will write $\Sigma+t$ for the hypersurface that results from "moving $\Sigma$ forward in time by $t$ units"; and if $\Delta$ is a subset of $\Sigma$, we will use $\Delta+t$ to denote the corresponding subset of $\Sigma+t$. We assume that there is a representation $t \mapsto U_{t}$ of the time-translation group $\mathbb{R}$ in the unitary operators on $\mathcal{H}$, and we will say that the localization system $\left(\mathcal{H}, \Delta \mapsto E_{\Delta}, t \mapsto U_{t}\right)$ satisfies time-translation covariance just in case $U_{t} E_{\Delta} U_{-t}=E_{\Delta+t}$ for all $\Delta$ and all $t \in \mathbb{R}$.

Hegerfeldt's result is based on the following root lemma. 
Lemma 1 (Hegerfeldt). Suppose that $U_{t}=e^{i t H}$, where $H$ is a self-adjoint operator with spectrum bounded from below. Let $A$ be a positive operator (e.g., a projection operator). Then for any state $\psi$, either

$$
\left\langle U_{t} \psi, A U_{t} \psi\right\rangle \neq 0, \quad \text { for almost all } t \in \mathbb{R},
$$

or

$$
\left\langle U_{t} \psi, A U_{t} \psi\right\rangle=0, \quad \text { for all } t \in \mathbb{R} .
$$

Hegerfeldt claims that this lemma has the following consequence for localization:

If there exist particle states which are strictly localized in some finite region at $t=0$ and later move towards infinity, then finite propagation speed cannot hold for localization of particles. (Hegerfeldt 1998a, 243)

Hegerfeldt's argument for this conclusion is as follows:

Now, if the particle or system is strictly localized in $\Delta$ at $t=0$ it is, a fortiori, also strictly localized in any larger region $\Delta^{\prime}$ containing $\Delta$. If the boundaries of $\Delta^{\prime}$ and $\Delta$ have a finite distance and if finite propagation speed holds then the probability to find the system in $\Delta^{\prime}$ must also be 1 for sufficiently small times, e.g. $0 \leq t<\epsilon$. But then [Lemma1], with $A \equiv I-E_{\Delta^{\prime}}$, states that the system stays in $\Delta^{\prime}$ for all times. Now, we can make $\Delta^{\prime}$ smaller and let it approach $\Delta$. Thus we conclude that if a particle or system is at time $t=0$ strictly localized in a region $\Delta$, then finite propagation speed implies that it stays in $\Delta$ for all times and therefore prohibits motion to infinity. (Hegerfeldt 1998a, 242-243; notation adapted, but italics in original)

Let us attempt now to put this argument into a more precise form.

First, Hegerfeldt claims that the following is a consequence of "finite propagation speed": If $\Delta \subseteq \Delta^{\prime}$, and if the boundaries of $\Delta$ and $\Delta^{\prime}$ have a finite distance, then a state initially localized in $\Delta$ will continue to be localized in $\Delta^{\prime}$ for some finite amount of time. We can capture this precisely by means of the following condition.

No instantaneous wavepacket spreading (NIWS): If $\Delta \subseteq \Delta^{\prime}$, and the boundaries of $\Delta$ and $\Delta^{\prime}$ have a finite distance, then there is an $\epsilon>0$ such that $E_{\Delta} \leq$ $E_{\Delta^{\prime}+t}$ whenever $0 \leq t<\epsilon$. 
(Note that NIWS plus localizability entails strong causality.) In the argument, Hegerfeldt also assumes that if a particle is localized in every one of a family of sets that "approaches" $\Delta$, then it is localized in $\Delta$. We can capture this assumption in the following condition.

Monotonicity: If $\left\{\Delta_{n}: n \in \mathbb{N}\right\}$ is a downward nested family of subsets of $\Sigma$ such that $\bigcap_{n} \Delta_{n}=\Delta$, then $\bigwedge_{n} E_{\Delta_{n}}=E_{\Delta}$.

Using this assumption, Hegerfeldt argues that if NIWS holds, and if a particle is initially localized in some finite region $\Delta$, then it will remain in $\Delta$ for all subsequent times. In other words, if $E_{\Delta} \psi=\psi$, then $E_{\Delta} U_{t} \psi=U_{t} \psi$ for all $t \geq 0$. We can now translate this into the following rigorous no-go theorem.

Theorem (Hegerfeldt). Suppose that the localization system $\left(\mathcal{H}, \Delta \mapsto E_{\Delta}, t \mapsto\right.$ $\left.U_{t}\right)$ satisfies:

\section{Monotonicity}

\section{Time-translation covariance}

\section{Energy bounded below}

\section{No instantaneous wavepacket spreading}

Then $U_{t} E_{\Delta} U_{-t}=E_{\Delta}$ for all $\Delta \subset \Sigma$ and all $t \in \mathbb{R}$.

(For the proof of this theorem, see Appendix A.)

Thus, conditions 1-4 can be satisfied only if the particle has trivial dynamics. If $M$ is an affine space, and if we add "no absolute velocity" as a fifth condition in this theorem, then we get the stronger conclusion that $E_{\Delta}=0$ for all bounded $\Delta$ (see Lemma 2, appendix). Thus, there is an obvious similarity between Hegerfeldt's and Malament's theorems. However, NIWS is a stronger causality assumption than microcausality. In fact, while NIWS plus localizability entails strong causality (and hence microcausality), the following example shows that NIWS is not entailed by the conjunction of strong causality, monotonicity, time-translation covariance, and energy bounded below.

Example 3. Let $Q, P$ denote the standard position and momentum operators on $\mathcal{H}=L_{2}(\mathbb{R})$, and let $H=P^{2} / 2 m$ for some $m>0$. Let $\Delta \mapsto E_{\Delta}^{Q}$ denote the spectral measure for $Q$. Fix some bounded subset $\Delta_{0}$ of $\mathbb{R}$, and let $E_{\Delta}=$ $E_{\Delta}^{Q} \otimes E_{\Delta_{0}}^{Q}$ (a projection operator on $\mathcal{H} \otimes \mathcal{H}$ ) for all Borel subsets $\Delta$ of $\mathbb{R}$. Thus, $\Delta \mapsto E_{\Delta}$ is a (non-normalized) projection-valued measure. Let $U_{t}=I \otimes e^{i t H}$, and let $E_{\Delta+t}=U_{t} E_{\Delta} U_{-t}$ for all $t \in \mathbb{R}$. It is clear that monotonicity, time-translation 
covariance, and energy bounded below hold. To see that strong causality holds, let $\Delta$ and $\Delta^{\prime}$ be disjoint subsets of a single hyperplane $\Sigma$. Then,

$$
E_{\Delta} U_{t} E_{\Delta^{\prime}} U_{-t}=E_{\Delta}^{Q} E_{\Delta^{\prime}}^{Q} \otimes E_{\Delta_{0}}^{Q} E_{\Delta_{0}+t}^{Q}=0 \otimes E_{\Delta}^{Q} E_{\Delta_{0}+t}^{Q}=0,
$$

for all $t \in \mathbb{R}$. On the other hand, $U_{t} E_{\Delta} U_{-t} \neq E_{\Delta}$ for any nonempty $\Delta$ and for any $t \neq 0$. Thus, it follows from Hegerfeldt's theorem that NIWS fails.

Thus, we could not recapture the full strength of Malament's theorem simply by adding "no absolute velocity" to the conditions of Hegerfeldt's theorem.

\section{A Strengthened Hegerfeldt-Malament Theorem}

Example 3 shows that Hegerfeldt's theorem fails if NIWS is replaced by strong causality (or by microcausality). On the other hand, Example 3 is hardly a physically interesting counterexample to a strengthened version of Hegerfeldt's theorem. In particular, if $\Sigma$ is a fixed spatial hypersurface, and if $\left\{\Delta_{n}: n \in \mathbb{N}\right\}$ is a covering of $\Sigma$ by bounded sets (i.e., $\bigcup_{n} \Delta_{n}=\Sigma$ ), then $\bigvee_{n} E_{\Delta_{n}}=I \otimes E_{\Delta_{0}} \neq I \otimes I$. Thus, it is not certain that the particle will be detected somewhere or other in space. In fact, if $\left\{\Delta_{n}: n \in \mathbb{N}\right\}$ is a covering of $\Sigma$ and $\left\{\Pi_{n}: n \in \mathbb{N}\right\}$ is a covering of $\Sigma+t$, then

$$
\bigvee_{n \in \mathbb{N}} E_{\Delta_{n}}=I \otimes E_{\Delta_{0}} \neq I \otimes E_{\Delta_{0}+t}=\bigvee_{n \in \mathbb{N}} E_{\Pi_{n}}
$$

Thus, the total probability for finding the particle somewhere or other in space can change over time.

It would be completely reasonable to require that $\bigvee_{n} E_{\Delta_{n}}=I$ whenever $\left\{\Delta_{n}\right.$ : $n \in \mathbb{N}\}$ is a covering of $\Sigma$. This would be the case, for example, if the mapping $\Delta \mapsto E_{\Delta}$ (restricted to subsets of $\Sigma$ ) were the spectral measure of some position operator. However, we propose that—at the very least—any physically interesting model should satisfy the following weaker condition.

Probability conservation: If $\left\{\Delta_{n}: n \in \mathbb{N}\right\}$ is a covering of $\Sigma$, and $\left\{\Pi_{n}: n \in \mathbb{N}\right\}$ is a covering of $\Sigma+t$, then $\bigvee_{n} E_{\Delta_{n}}=\bigvee_{n} E_{\Pi_{n}}$.

Probability conservation guarantees that there is a well-defined total probability for finding the particle somewhere or other in space, and this probability remains constant over time. In particular, if both $\left\{\Delta_{n}: n \in \mathbb{N}\right\}$ and $\left\{\Pi_{n}: n \in \mathbb{N}\right\}$ consist of pairwise disjoint sets, then the localizability condition entails that $\bigvee_{n} E_{\Delta_{n}}=$ $\sum_{n} E_{\Delta_{n}}$ and $\bigvee_{n} E_{\Pi_{n}}=\sum_{n} E_{\Pi_{n}}$. In this case, probability conservation is equivalent to

$$
\sum_{n \in \mathbb{N}} \operatorname{Prob}^{\psi}\left(E_{\Delta_{n}}\right)=\sum_{n \in \mathbb{N}} \operatorname{Prob}^{\psi}\left(E_{\Pi_{n}}\right)
$$


for any state $\psi$. Note, finally, that probability conservation is neutral with respect to relativistic and non-relativistic models."]

Theorem 1 (Strengthened Hegerfeldt-Malament Theorem). Suppose that the localization system $\left(\mathcal{H}, \Delta \mapsto E_{\Delta}, t \mapsto U_{t}\right)$ satisfies:

\section{Localizability}

\section{Probability conservation}

\section{Time-translation covariance}

\section{Energy bounded below}

\section{Microcausality}

Then $U_{t} E_{\Delta} U_{-t}=E_{\Delta}$ for all $\Delta$ and all $t \in \mathbb{R}$.

(For the proof of this theorem, see Appendix A.)

If $M$ is an affine space, and if we add "no absolute velocity" as a sixth condition in this theorem, then it follows that $E_{\Delta}=0$ for all $\Delta$ (see Lemma2). Thus, modulo the probability conservation condition, Theorem 1 recaptures the full strength of Malament's theorem. Moreover, we can now trace the difficulties with localization to microcausality alone: there are localizable particles only if it is possible to have act-outcome correlations at spacelike separation.

We now give examples to show that each condition in Theorem 11 is indispensable; that is, no four of the conditions suffices to entail the conclusion. (Example 1 shows that conditions 1-5 can be simultaneously satisfied.) Suppose for simplicity that $M$ is two-dimensional. (All examples work in the four-dimensional case as well.) Let $Q, P$ be the standard position and momentum operators on $L_{2}(\mathbb{R})$, and let $H=P^{2} / 2 m$. Let $\Sigma$ be a spatial hypersurface in $M$, and suppose that a coordinatization of $\Sigma$ has been fixed, so that there is a natural association between each bounded open subset $\Delta$ of $\Sigma$ and a corresponding spectral projection $E_{\Delta}$ of $Q$.

$(1+2+3+4)$ (a) Consider the standard localization system for a single non-relativistic particle. That is, let $\Sigma$ be a fixed spatial hyperplane, and let $\Delta \mapsto E_{\Delta}$ (with domain the Borel subsets of $\Sigma$ ) be the spectral measure for $Q$. For $\Sigma+t$, set $E_{\Delta+t}=U_{t} E_{\Delta} U_{-t}$, where $U_{t}=e^{i t H}$. (b) The Newton-Wigner approach to relativistic $\mathrm{QM}$ uses the standard localization system for a non-relativistic

\footnotetext{
${ }^{1}$ Probability conservation would fail if a particle could escape to infinity in a finite amount of time (cf. Earman 1986, 33). However, a particle can escape to infinity only if there is an infinite potential well, and this would violate the energy condition. Thus, given the energy condition, probability conservation should also hold for non-relativistic particle theories.
} 
particle, only replacing the non-relativistic Hamiltonian $P^{2} / 2 m$ with the relativistic Hamiltonian $\left(P^{2}+m^{2} I\right)^{1 / 2}$, whose spectrum is also bounded from below.

$(1+2+3+5)$ (a) For a mathematically simple (but physically uninteresting) example, take the first example above and replace the Hamiltonian $P^{2} / 2 m$ with $P$. In this case, microcausality trivially holds, since $U_{t} E_{\Delta} U_{-t}$ is just a shifted spectral projection of $Q$. (b) For a physically interesting example, consider the relativistic quantum theory of a single spin- $1 / 2$ electron (see Section 2). Due to the negative energy solutions of the Dirac equation, the spectrum of the Hamiltonian is not bounded from below.

$(1+2+4+5)$ Consider the the standard localization system for a non-relativistic particle, but set $E_{\Delta+t}=E_{\Delta}$ for all $t \in \mathbb{R}$. Thus, we escape the conclusion of trivial dynamics, but only by disconnecting the (nontrivial) unitary dynamics from the (trivial) association of projections with spatial regions.

$(1+3+4+5)$ (a) Let $\Delta_{0}$ be some bounded open subset of $\Sigma$, and let $E_{\Delta_{0}}$ be the corresponding spectral projection of $Q$. When $\Delta \neq \Delta_{0}$, let $E_{\Delta}=0$. Let $U_{t}=e^{i t H}$, and let $E_{\Delta+t}=U_{t} E_{\Delta} U_{-t}$ for all $\Delta$. This example is physically uninteresting, since the particle cannot be localized in any region besides $\Delta_{0}$, including proper supersets of $\Delta_{0}$. (b) See Example 3 .

$(2+3+4+5)$ Let $\Delta_{0}$ be some bounded open subset of $\Sigma$, and let $E_{\Delta_{0}}$ be the corresponding spectral projection of $Q$. When $\Delta \neq \Delta_{0}$, let $E_{\Delta}=I$. Let $U_{t}=e^{i t H}$, and let $E_{\Delta+t}=U_{t} E_{\Delta} U_{-t}$ for all $\Delta$. Thus, the particle is always localized in every region other than $\Delta_{0}$, and is sometimes localized in $\Delta_{0}$ as well.

\section{Are there Unsharply Localizable Particles?}

We have argued that attempts to undermine the four explicit assumptions of Malament's theorem are unsuccessful. We have also now shown that the tacit assumption of "no absolute velocity" is not necessary to derive Malament's conclusion. And, yet, there is one more loophole in the argument against a relativistic quantum mechanics of localizable particles. In particular, the basic assumption of a family $\left\{E_{\Delta}\right\}$ of localizing projections is unnecessary; it is possible to have a quantummechanical particle theory in the absence of localizing projections. What is more, one might object to the use of localizing projections on the grounds that they represent an unphysical idealization-viz., that a "particle" can be completely contained in a finite region of space with a sharp boundary, when in fact it would require an 
infinite amount of energy to prepare a particle in such a state. Thus, there remains a possibility that relativistic causality can be reconciled with "unsharp" localizability.

To see how we can define "particle talk" without having projection operators, consider the relativistic theory of a single spin- $1 / 2$ electron (where we now restrict to the subspace $\mathcal{H}_{\text {pos }}$ of positive energy solutions of the Dirac equation). In order to treat the ' $x$ ' of the Dirac wavefunction as an observable, we need only to define a probability amplitude and density for the particle to be found at $\boldsymbol{x}$; and these can be obtained from the Dirac wavefunction itself. That is, for a subset $\Delta$ of $\Sigma$, we set

$$
\operatorname{Prob}^{\psi}(\boldsymbol{x} \in \Delta)=\int_{\Delta}|\psi(\boldsymbol{x})|^{2} d \boldsymbol{x} .
$$

Now let $\Delta \mapsto E_{\Delta}$ be the spectral measure for the standard position operator on the Hilbert space $\mathcal{H}$ (which includes both positive and negative energy solutions). That is, $E_{\Delta}$ multiplies a wavefunction by the characteristic function of $\Delta$. Let $F$ denote the orthogonal projection of $\mathcal{H}$ onto $\mathcal{H}_{\text {pos }}$. Then,

$$
\int_{\Delta}|\psi(\boldsymbol{x})|^{2} d \boldsymbol{x}=\left\langle\psi, E_{\Delta} \psi\right\rangle=\left\langle\psi, F E_{\Delta} \psi\right\rangle,
$$

for any $\psi \in \mathcal{H}_{\text {pos }}$. Thus, we can apply the standard recipe to the operator $F E_{\Delta}$ (defined on $\mathcal{H}_{\text {pos }}$ ) to compute the probability that the particle will be found within $\Delta$. However, $F E_{\Delta}$ does not define a projection operator on $\mathcal{H}_{\text {pos. }}$ (In fact, it can be shown that $F E_{\Delta}$ does not have any eigenvectors with eigenvalue 1.) Thus, we do not need a family of projection operators in order to define probabilities for localization.

Now, in general, to define the probability that a particle will be found in $\Delta$, we need only assume that there is an operator $A_{\Delta}$ such that $\left\langle\psi, A_{\Delta} \psi\right\rangle \in[0,1]$ for any unit vector $\psi$. Such operators are called effects, and include the projection operators as a proper subclass. Thus, we say that the triple $\left(\mathcal{H}, \Delta \mapsto A_{\Delta}, \mathbf{a} \mapsto U(\mathbf{a})\right)$ is an unsharp localization system over $M$ just in case $\Delta \mapsto A_{\Delta}$ is a mapping from subsets of hyperplanes in $M$ to effects on $\mathcal{H}$, and $\mathbf{a} \mapsto U(\mathbf{a})$ is a continuous representation of the translation group of $M$ in unitary operators on $\mathcal{H}$. (We assume for the present that $M$ is again an affine space.)

Most of the conditions from the previous sections can be applied, with minor changes, to unsharp localization systems. In particular, since the energy bounded below condition refers only to the unitary representation, it can be carried over intact; and translation covariance also generalizes straightforwardly. However, we will need to take more care with microcausality and with localizability.

If $E$ and $F$ are projection operators, $[E, F]=0$ just in case for any state, the statistics of a measurement of $F$ are not affected by a non-selective measurement 
of $E$ and vice versa (cf. Malament 1996, 5). This fact, along with the assumption that $E_{\Delta}$ is measurable in $\Delta$, motivates the microcausality assumption. For the case of an association of arbitrary effects with spatial regions, Busch (1999, Proposition 2 ) has shown that $\left[A_{\Delta}, A_{\Delta^{\prime}}\right]=0$ just in case for any state, the statistics for a measurement of $A_{\Delta}$ are not affected by a non-selective measurement of $A_{\Delta^{\prime}}$ and vice versa. Thus, we may carry over the microcausality assumption intact, again seen as enforcing a prohibition against act-outcome correlations at spacelike separation.

The localizability condition is motivated by the idea that a particle cannot be simultaneously localized (with certainty) in two disjoint regions of space. In other words, if $\Delta$ and $\Delta^{\prime}$ are disjoint subsets of a single hyperplane, then $\left\langle\psi, E_{\Delta} \psi\right\rangle=1$ entails that $\left\langle\psi, E_{\Delta^{\prime}} \psi\right\rangle=0$. It is not difficult to see that this last condition is equivalent to the assumption that $E_{\Delta}+E_{\Delta^{\prime}} \leq I$. That is,

$$
\left\langle\psi,\left(E_{\Delta}+E_{\Delta^{\prime}}\right) \psi\right\rangle \leq\langle\psi, I \psi\rangle,
$$

for any state $\psi$. Now, it is an accidental feature of projection operators (as opposed to arbitrary effects) that $E_{\Delta}+E_{\Delta^{\prime}} \leq I$ is equivalent to $E_{\Delta} E_{\Delta^{\prime}}=0$. Thus, the apropriate generalization of localizability to unsharp localization systems is the following condition.

Localizability: If $\Delta$ and $\Delta^{\prime}$ are disjoint subsets of a single hyperplane, then

$$
A_{\Delta}+A_{\Delta^{\prime}} \leq I \text {. }
$$

That is, the probability for finding the particle in $\Delta$, plus the probability for finding the particle in some disjoint region $\Delta^{\prime}$, never totals more than 1 . It would, in fact, be reasonable to require a slightly stronger condition, viz., the probability of finding a particle in $\Delta$ plus the probability of finding a particle in $\Delta^{\prime}$ equals the probability of finding a particle in $\Delta \cup \Delta^{\prime}$. If this is true for all states $\psi$, we have:

Additivity: If $\Delta$ and $\Delta^{\prime}$ are disjoint subsets of a single hyperplane, then

$$
A_{\Delta}+A_{\Delta^{\prime}}=A_{\Delta \cup \Delta^{\prime}}
$$

With just these mild constraints, Busch (1999) was able to derive the following no-go result.

Theorem (Busch). Suppose that the unsharp localization system $\left(\mathcal{H}, \Delta \mapsto A_{\Delta}, \mathbf{a} \mapsto\right.$ $U(\mathbf{a}))$ satisfies localizability, translation covariance, energy bounded below, microcausality, and no absolute velocity. Then, for all $\Delta, A_{\Delta}$ has no eigenvector with eigenvalue 1 .

Thus, it is not possible for a particle to be localized with certainty in any bounded region $\Delta$. Busch's theorem, however, leaves it open question whether there are (nontrivial) "strongly unsharp" localization systems that satisfy microcausality. The following result shows that there are not. 
Theorem 2. Suppose that the unsharp localization system $\left(\mathcal{H}, \Delta \mapsto A_{\Delta}, \mathbf{a} \mapsto\right.$ $U(\mathbf{a}))$ satisfies:

\section{Additivity}

\section{Translation covariance}

\section{Energy bounded below}

\section{Microcausality}

\section{No absolute velocity}

Then $A_{\Delta}=0$ for all $\Delta$.

(For the proof of this theorem, see Appendix B.)

Theorem 2 shows that invoking the notion of unsharp localization does nothing to resolve the tension between relativistic causality and localizability. For example, we can now show that the (positive energy) Dirac theory-in which there are localizable particles-violates relativistic causality. Indeed, it is clear that the conclusion of Theorem 1 fails. [f On the other hand, additivity, translation covariance, energy bounded below, and no absolute velocity hold. Thus, microcausality fails, and the (positive energy) Dirac theory permits superluminal signalling.

Unfortunately, Theorem 2 does not generalize to arbitrary globally hyperbolic spacetimes, as the following example shows.

Example 4. Let $M$ be the cylinder spacetime from Example 2. Let $G$ denote the group of timelike translations and rotations of $M$, and let $g \mapsto U(g)$ be a positive energy representation of $G$ in the unitary operators on a Hilbert space $\mathcal{H}$. For any $\Sigma \in \mathcal{S}$, let $\mu$ denote the normalized rotation-invariant measure on $\Sigma$, and let $A_{\Delta}=\mu(\Delta) I$. Then, conditions 1-5 of Theorem $⿴$ are satisfied, but the conclusion of the theorem is false.

The previous counterexample can be excluded if we require there to be a fixed positive constant $\delta$ such that, for each $\Delta$, there is a state $\psi$ with $\left\langle\psi, A_{\Delta} \psi\right\rangle \geq \delta$. In fact, with this condition added, Theorem 2 holds for any globally hyperbolic spacetime. (The proof is an easy modification of the proof we give in Appendix B.) However, it is not clear what physical motivation there could be for requiring this further condition. Note also that Example $\bigoplus$ has trivial dynamics; i.e., $U_{t} A_{\Delta} U_{-t}=$ $A_{\Delta}$ for all $\Delta$. We conjecture that every counterexample to a generalized version of Theorem 2 will have trivial dynamics.

\footnotetext{
${ }^{2}$ For any unit vector $\psi \in \mathcal{H}_{\text {pos }}$, there is a bounded set $\Delta$ such that $\int_{\Delta}|\psi|^{2} d \boldsymbol{x} \neq 0$. Thus, $A_{\Delta} \neq 0$.
} 
Theorem 2 strongly supports the conclusion that there is no relativistic quantum mechanics of a single (localizable) particle; and that the only consistent combination of special relativity and quantum mechanics is in the context of quantum field theory. However, neither Theorem 1 nor Theorem 2 says anything about the ontology of relativistic quantum field theory itself; they leave open the possibility that relativistic quantum field theory might permit an ontology of localizable particles. To eliminate this latter possibility, we will now proceed to present a more general result which shows that there are no localizable particles in any relativistic quantum theory.

\section{Are there Localizable Particles in RQFT?}

The localizability assumption is motivated by the idea that a "particle" cannot be detected in two disjoint spatial regions at once. However, in the case of a manyparticle system, it is certainly possible for there to be particles in disjoint spatial regions. Thus, the localizability condition does not apply to many-particle systems; and Theorems 1 and 2 cannot be used to rule out a relativistic quantum mechanics of $n>1$ localizable particles.

Still, one might argue that we could use $E_{\Delta}$ to represent the proposition that a measurement is certain to find that all $n$ particles lie within $\Delta$, in which case localizability should hold. Note, however, that when we alter the interpretation of the localization operators $\left\{E_{\Delta}\right\}$, we must alter our interpretation of the conclusion. In particular, the conclusion now shows only that it is not possible for all $n$ particles to be localized in a bounded region of space. This leaves open the possibility that there are localizable particles, but that they are governed by some sort of "exclusion principle" that prohibits them all from clustering in a bounded spacetime region.

Furthermore, Theorems 1 and 0 only show that it is impossible to define position operators that obey appropriate relativistic constraints. But it does not immediately follow from this that we lack any notion of localization in relativistic quantum theories. Indeed,

.... position operator is inconsistent with relativity. This compels us to find another way of modeling localization of events. In field theory, we model localization by making the observables dependent on position in spacetime. (Ticiatti 1999, 11)

However, it is not a peculiar feature of relativistic quantum field theory that it lacks a position operator: Any quantum field theory (either relativistic or non-relativistic) will model localization by making the observables dependent on position in spacetime. Moreover, in the case of non-relativistic QFT, these "localized" observables 
suffice to provide us with a concept of localizable particles. In particular, for each spatial region $\Delta$, there is a "number operator" $N_{\Delta}$ whose eigenvalues give the number of particles within the region $\Delta$. Thus, we have no difficultly in talking about the particle content in a given region of space despite the absence of any position operator.

Abstractly, a number operator $N$ on $\mathcal{H}$ is any operator with eigenvalues contained in $\{0,1,2, \ldots\}$. In order to describe the number of particles locally, we require an association $\Delta \mapsto N_{\Delta}$ of subsets of spatial hyperplanes in $M$ to number operators on $\mathcal{H}$, where $N_{\Delta}$ represents the number of particles in the spatial region $\Delta$. If $\mathbf{a} \mapsto U(\mathbf{a})$ is a unitary representation of the translation group, we say that the triple $\left(\mathcal{H}, \Delta \mapsto N_{\Delta}, \mathbf{a} \mapsto U(\mathbf{a})\right)$ is a system of local number operators over $M$. Note that a localization system $\left(\mathcal{H}, \Delta \mapsto E_{\Delta}, \mathbf{a} \mapsto U(\mathbf{a})\right)$ is a special case of a system of local number operators where the eigenvalues of each $N_{\Delta}$ are restricted to $\{0,1\}$. Furthermore, if we loosen our assumption that number operators have a discrete spectrum, and instead require only that they have spectrum contained in $[0, \infty)$, then we can also include unsharp localization systems within the general category of systems of local number operators. Thus, a system of local number operators is the minimal requirement for a concept of localizable particles in any quantum theory.

In addition to the natural analogues of the energy bounded below condition, translation covariance, and microcausality, we will be interested in the following two requirements on a system of local number operators:[]

Additivity: If $\Delta$ and $\Delta^{\prime}$ are disjoint subsets of a single hyperplane, then

$$
N_{\Delta}+N_{\Delta^{\prime}}=N_{\Delta \cup \Delta^{\prime}} .
$$

Number conservation: If $\left\{\Delta_{n}: n \in \mathbb{N}\right\}$ is a disjoint covering of $\Sigma$, then the sum $\sum_{n} N_{\Delta_{n}}$ converges to a densely defined, self-adjoint operator $N$ on $\mathcal{H}$ (independent of the chosen covering), and $U(\mathbf{a}) N U(\mathbf{a})^{*}=N$ for any timelike translation a of $M$.

Additivity asserts that, when $\Delta$ and $\Delta^{\prime}$ are disjoint, the expectation value (in any state $\psi$ ) for the number of particles in $\Delta \cup \Delta^{\prime}$ is the sum of the expectations for the number of particles in $\Delta$ and the number of particles in $\Delta^{\prime}$. In the pure case, it asserts that the number of particles in $\Delta \cup \Delta^{\prime}$ is the sum of the number of particles

\footnotetext{
${ }^{3}$ Due to the unboundedness of number operators, we would need to take some care in giving technically correct versions of the following conditions. In particular, the additivity condition should technically include the clause that $N_{\Delta}$ and $N_{\Delta^{\prime}}$ have a common dense domain, and the operator $N_{\Delta \cup \Delta^{\prime}}$ should be thought of as the self-adjoint closure of $N_{\Delta}+N_{\Delta^{\prime}}$. In the number conservation condition, the sum $N=\sum_{n} N_{\Delta_{n}}$ can be made rigorous by exploiting the correspondence between self-adjoint operators and "quadratic forms" on $\mathcal{H}$. In particular, we can think of $N$ as deriving from the upper bound of quadratic forms corresponding to finite sums.
} 
in $\Delta$ and the number of particles in $\Delta^{\prime}$. The "number conservation" condition tells us that there is a well-defined total number of particles (at a given time), and that the total number of particles does not change over time. This condition holds for any non-interacting model of QFT.

It is a well-known consequence of the Reeh-Schlieder theorem that relativistic quantum field theories do not admit systems of local number operators (cf. Redhead 1995). We will now derive the same conclusion from strictly weaker assumptions. In particular, we show that microcausality is the only specifically relativistic assumption needed for this result. The relativistic spectrum condition-which requires that the spectrum of the four-momentum lie in the forward light cone, and which is used in the proof of the Reeh-Schlieder theorem-plays no role in our proof.t.

Theorem 3. Suppose that the system $\left(\mathcal{H}, \Delta \mapsto N_{\Delta}, \mathbf{a} \mapsto U(\mathbf{a})\right)$ of local number operators satisfies:

\section{Additivity}

\section{Translation covariance}

3. Energy bounded below

\section{Number conservation}

\section{Microcausality}

6. No absolute velocity

Then $N_{\Delta}=0$ for all $\Delta$.

(For the proof of the theorem, see Appendix C.)

Thus, in every state, there are no particles in any local region. This serves as a reductio ad absurdum for any notion of localizable particles in a relativistic quantum theory.

Unfortunately, Theorem 3 is not the strongest result we could hope for, since "number conservation" can only be expected to hold in the (trivial) case of noninteracting fields. However, we would need a more general approach in order to deal with interacting relativistic quantum fields, because (due to Haag's theorem; cf. Streater and Wightman, 2000, 163) their dynamics are not unitarily implementable on a fixed Hilbert space. On the other hand it would be wrong to think of

\footnotetext{
${ }^{4}$ Microcausality is not only sufficient, but also necessary for the proof that there are no local number operators. The Reeh-Schlieder theorem entails the cyclicity of the vacuum state. But the cyclicity of the vacuum state alone does not entail that there are no local number operators; we must also assume microcausality (cf. Halvorson 2001, Requardt 1982).
} 
this as indicating a limitation on the generality of our conclusion: Haag's theorem also entails that interacting models of RQFT have no number operators-either global or local.[ Still, it would be interesting to recover this conclusion (perhaps working in a more general algebraic setting) without using the full strength of Haag's assumptions.

\section{Particle Talk without Particle Ontology}

The results of the previous sections show that, insofar as we can expect any relativistic quantum theory theory to satisfy a few basic conditions, these theories do not admit (localizable) particles into their ontology. We also considered and rejected several arguments which attempt to show that one (or more) of these conditions can be jettisoned without doing violence to the theory of relativity or to quantum mechanics. Thus, we have yet to find a good reason to reject one of the premises on which our argument against localizable particles is based. However, Segal (1964) and Barrett (2000) claim that we have independent grounds for rejecting the conclusion; that is, we have good reasons for believing that there are localizable particles.

The argument for localizable particles appears to be very simple: Our experience shows us that objects (particles) occupy finite regions of space. But the reply to this argument is just as simple: These experiences are illusory! Although no object is strictly localized in a bounded region of space, an object can be well-enough localized to give the appearance to us (finite observers) that it is strictly localized. In fact, relativistic quantum field theory itself shows how the "illusion" of localizable particles can arise, and how talk about localizable particles can be a useful fiction.

In order to assess the possibility of "approximately localized" objects in relativistic quantum field theory, we shall now pursue the investigation in the framework of algebraic quantum field theory. respondence $\mathcal{O} \mapsto R(\mathcal{O})$ between bounded open subsets of $M$ and subalgebras of observables on some Hilbert space $\mathcal{H}$. Observables in $R(\mathcal{O})$ are considered to be "localized" (i.e., measurable) in $\mathcal{O}$. Thus, if $\mathcal{O}$ and $\mathcal{O}^{\prime}$ are spacelike separated, we require that $[A, B]=0$ for any $A \in R(\mathcal{O})$ and $B \in R\left(\mathcal{O}^{\prime}\right)$. Furthermore,

\footnotetext{
${ }^{5}$ If a total number operator exists in a representation of the canonical commutation relations, then that representation is quasiequivalent to a free-field (Fock) representation (Chaiken 1968). However, Haag's theorem entails that in relativistic theories, representations with nontrivial interactions are not quasiequivalent to a free-field representation.

${ }^{6}$ For general information on algebraic quantum field theory, see (Haag 1992) and (Buchholz 2000). For specific information on particle detectors and "almost local" observables, see Chapter 6 of (Haag 1992) and Section 4 of (Buchholz 2000).
} 
we assume that there is a continuous representation $\mathbf{a} \mapsto U(\mathbf{a})$ of the translation group of $M$ in unitary operators on $\mathcal{H}$, and that there is a unique "vacuum" state $\Omega \in \mathcal{H}$ such that $U(\mathbf{a}) \Omega=\Omega$ for all a. This latter condition entails that the vacuum appears the same to all observers, and that it is the unique state of lowest energy.

In this context, a particle detector can be represented by an effect $C$ such that $\langle\Omega, C \Omega\rangle=0$. That is, $C$ should register no particles in the vacuum state. However, the Reeh-Schlieder theorem entails that no positive local observable can have zero expectation value in the vacuum state. Thus, we again see that (strictly speaking) it is impossible to detect particles by means of local measurements; instead, we will have to think of particle detections as "approximately local" measurements.

If we think of an observable as representing a measurement procedure (or, more precisely, an equivalence class of measurement procedures), then the norm distance $\left\|C-C^{\prime}\right\|$ between two observables gives a quantitative measure of the physical similarity between the corresponding procedures. (In particular, if $\left\|C-C^{\prime}\right\|<\delta$, then the expectation values of $C$ and $C^{\prime}$ never differ by more than $\delta$.) $]$ Moreover, in the case of real-world measurements, the existence of measurement errors and environmental noise make it impossible for us to determine precisely which measurement procedure we have performed. Thus, practically speaking, we can at best determine a neighborhood of observables corresponding to a concrete measurement procedure.

In the case of present interest, what we actually measure is always a local observable-i.e., an element of $R(\mathcal{O})$, where $\mathcal{O}$ is bounded. However, given a fixed error bound $\delta$, if an observable $C$ is within norm distance $\delta$ from some local observable $C^{\prime} \in R(\mathcal{O})$, then a measurement of $C^{\prime}$ will be practically indistinguishable from a measurement of $C$. Thus, if we let

$$
R_{\delta}(\mathcal{O})=\left\{C: \exists C^{\prime} \in R(\mathcal{O}) \text { such that }\left\|C-C^{\prime}\right\|<\delta\right\},
$$

denote the family of observables "almost localized" in $\mathcal{O}$, then 'FAPP' (i.e., 'for all practical purposes') we can locally measure any observable from $R_{\delta}(\mathcal{O})$. That is, measurement of an element from $R_{\delta}(\mathcal{O})$ can be simulated to a high degree of accuracy by local measurement of an element from $R(\mathcal{O})$. However, for any local region $\mathcal{O}$, and for any $\delta>0, R_{\delta}(\mathcal{O})$ does contain (nontrivial) effects that annihilate the vacuum. . $^{3}$ Thus, particle detections can always be simulated by purely local measurements; and the appearance of (fairly-well) localized objects can be

\footnotetext{
${ }^{7}$ Recall that $\left\|C-C^{\prime}\right\|$ is defined as the supremum of $\left\|\left(C-C^{\prime}\right) \psi\right\|$ as $\psi$ runs through the unit vectors in $\mathcal{H}$. It follows, then, from the Cauchy-Schwarz inequality that $\left|\left\langle\psi,\left(C-C^{\prime}\right) \psi\right\rangle\right| \leq$ $\left\|C-C^{\prime}\right\|$ for any unit vector $\psi$.

${ }^{8}$ Suppose that $A \in R(\mathcal{O})$, and let $A(\boldsymbol{x})=U(\boldsymbol{x}) A U(\boldsymbol{x})^{*}$. If $f$ is a test function on $M$ whose Fourier transform is supported in the complement of the forward light cone, then $L=\int f(\boldsymbol{x}) A(\boldsymbol{x}) d \boldsymbol{x}$ is almost localized in $\mathcal{O}$ and $\langle\Omega, L \Omega\rangle=0$ (cf. Buchholz 2000, 7).
} 
explained without the supposition that there are localizable particles in the strict sense.

However, it may not be easy to pacify Segal and Barrett with a FAPP solution to the problem of localization. Both appear to think that the absence of localizable particles (in the strict sense) is not simply contrary to our manifest experience, but would undermine the very possiblity of objective empirical science. For example, Segal claims that,

...it is an elementary fact, without which experimentation of the usual sort would not be possible, that particles are indeed localized in space at a given time. (Segal 1965, 145; our italics)

Furthermore, "particles would not be observable without their localization in space at a particular time" $(1964,139)$. In other words, experimentation involves observations of particles, and these observations can occur only if particles are localized in space. Unfortunately, Segal does not give any argument for these claims. It seems to us, however, that the moral we should draw from the no-go theorems is that Segal's account of observation is false. In particular, it is not (strictly speaking) true that we observe particles. Rather, there are 'observation events', and these observation events are consistent (to a good degree of accuracy) with the supposition that they are brought about by (localizable) particles.

Like Segal, Barrett (2000) claims that we will have trouble explaining how empirical science can work if there are no localizable particles. In particular, Barrett claims that empirical science requires that we be able to keep an account of our measurement results so that we can compare these results with the predictions of our theories. Furthermore, we identify measurement records by means of their location in space. Thus, if there were no localized objects, then there would be no identifiable measurement records, and "...it would be difficult to account for the possibility of empirical science at all" (Barrett 2000, 3).

However, it's not clear what the difficulty here is supposed to be. On the one hand, we have seen that relativistic quantum field theory does predict that the appearances will be FAPP consistent with the supposition that there are localized objects. So, for example, we could distinguish two record tokens at a given time if there were two disjoint regions $\mathcal{O}$ and $\mathcal{O}^{\prime}$ and particle detector observables $C \in R_{\delta}(\mathcal{O})$ and $C^{\prime} \in R_{\delta}\left(\mathcal{O}^{\prime}\right)$ (approximated by observables strictly localized in $\mathcal{O}$ and $\mathcal{O}$ respectively) such that $\langle\psi, C \psi\rangle \approx 1$ and $\left\langle\psi, C^{\prime} \psi\right\rangle \approx 1$. Now, it may be that Barrett is also worried about how, given a field ontology, we could assign any sort of trans-temporal identity to our record tokens. But this problem, however important philosophically, is distinct from the problem of localization. Indeed, it also arises in the context of non-relativistic quantum field theory, where there is no problem with describing localizable particles. Finally, Barrett might object that 
once we supply a quantum-theoretical model of a particle detector itself, then the superposition principle will prevent the field and detector from getting into a state where there is a fact of the matter as to whether, "a particle has been detected in the region $\mathcal{O}$." But this is simply a restatement of the standard quantum measurement problem that infects all quantum theories - and we have made no pretense of solving that here.

\section{Conclusion}

Malament claims that his theorem justifies the belief that,

...in the attempt to reconcile quantum mechanics with relativity theory...one is driven to a field theory; all talk about "particles" has to be understood, at least in principle, as talk about the properties of, and interactions among, quantized fields. (Malament 1996, 1)

We have argued that the first claim is correct—quantum mechanics and relativity can be reconciled only in the context of quantum field theory. In order, however, to close a couple of loopholes in Malament's argument for this conclusion, we provided two further results (Theorems 1 and 2 ) which show that the conclusion continues to hold for generic spacetimes, as well as for "unsharp" localization observables. We then went on to show that relativistic quantum field theory also does not permit an ontology of localizable particles; and so, strictly speaking, our talk about localizable particles is a fiction. Nonetheless, relativistic quantum field theory does permit talk about particles-albeit, if we understand this talk as really being about the properties of, and interactions among, quantized fields. Indeed, modulo the standard quantum measurement problem, relativistic quantum field theory has no trouble explaining the appearance of macroscopically well-localized objects, and shows that our talk of particles, though a façon de parler, has a legitimate role to play in empirically testing the theory.

Acknowledgments: We would like to thank Jeff Barrett and David Malament for helpful correspondence.

\section{A Appendix}

Theorem (Hegerfeldt). Suppose that the localization system $\left(\mathcal{H}, \Delta \mapsto E_{\Delta}, t \mapsto\right.$ $\left.U_{t}\right)$ satisfies monotonicity, time-translation covariance, energy bounded below, and NIWS. Then $U_{t} E_{\Delta} U_{-t}=E_{\Delta}$ for all $\Delta \subset \Sigma$ and all $t \in \mathbb{R}$. 
Proof. The formal proof corresponds directly to Hegerfeldt's informal proof. Thus, let $\Delta$ be a subset of some spatial hypersurface $\Sigma$. If $E_{\Delta}=0$ then obviously $U_{t} E_{\Delta} U_{-t}=E_{\Delta}$ for all $t \in \mathbb{R}$. So, suppose that $E_{\Delta} \neq 0$, and let $\psi$ be a unit vector such that $E_{\Delta} \psi=\psi$. Since $\Sigma$ is a manifold, and since $\Delta \neq \Sigma$, there is a family $\left\{\Delta_{n}: n \in \mathbb{N}\right\}$ of subsets of $\Sigma$ such that, for each $n \in \mathbb{N}$, the distance between the boundaries of $\Delta_{n}$ and $\Delta$ is nonzero, and such that $\bigcap_{n} \Delta_{n}=\Delta$. Fix $n \in \mathbb{N}$. By NIWS and time-translation covariance, there is an $\epsilon_{n}>0$ such that $E_{\Delta_{n}} U_{t} \psi=U_{t} \psi$ whenever $0 \leq t<\epsilon_{n}$. That is, $\left\langle U_{t} \psi, E_{\Delta_{n}} U_{t} \psi\right\rangle=1$ whenever $0 \leq t<\epsilon_{n}$. Since energy is bounded from below, we may apply Lemma 1 with $A=I-E_{\Delta_{n}}$ to conclude that $\left\langle U_{t} \psi, E_{\Delta_{n}} U_{t} \psi\right\rangle=1$ for all $t \in \mathbb{R}$. That is, $E_{\Delta_{n}} U_{t} \psi=U_{t} \psi$ for all $t \in \mathbb{R}$. Since this holds for all $n \in \mathbb{N}$, and since (by monotonicity) $E_{\Delta}=\bigwedge_{n} E_{\Delta_{n}}$, it follows that $E_{\Delta} U_{t} \psi=U_{t} \psi$ for all $t \in \mathbb{R}$. Thus, $U_{t} E_{\Delta} U_{-t}=E_{\Delta}$ for all $t \in \mathbb{R}$.

Lemma 2. Suppose that the localization system $\left(\mathcal{H}, \Delta \mapsto E_{\Delta}, \mathbf{a} \mapsto U(\mathbf{a})\right)$ satisfies localizability, time-translation covariance, and no absolute velocity. Let $\Delta$ be a bounded spatial set. If $U(\mathbf{a}) E_{\Delta} U(\mathbf{a})^{*}=E_{\Delta}$ for all timelike translations a of $M$, then $E_{\Delta}=0$.

Proof. By no absolute velocity, there is a pair $(\mathbf{a}, \mathbf{b})$ of timelike translations such that $\Delta+(\mathbf{a}-\mathbf{b})$ is in $\Sigma$ and is disjoint from $\Delta$. By time-translation covariance, we have,

$$
E_{\Delta+(\mathbf{a}-\mathbf{b})}=U(\mathbf{a}) U(\mathbf{b})^{*} E_{\Delta} U(\mathbf{b}) U(\mathbf{a})^{*}=E_{\Delta} .
$$

Thus, localizability entails that $E_{\Delta}$ is orthogonal to itself, and so $E_{\Delta}=0$.

Lemma 3. Let $\left\{\Delta_{n}: n=0,1,2, \ldots\right\}$ be a covering of $\Sigma$, and let $E=\bigvee_{n=0}^{\infty} E_{\Delta_{n}}$. If probability conservation and time-translation covariance hold, then $U_{t} E U_{-t}=$ $E$ for all $t \in \mathbb{R}$.

Proof. Since $\left\{\Delta_{n}+t: n \in \mathbb{N}\right\}$ is a covering of $\Sigma+t$, probability conservation entails that $\bigvee_{n} E_{\Delta_{n}+t}=E$. Thus,

$$
\begin{aligned}
U_{t} E U_{-t} & =U_{t}\left[\bigvee_{n=0}^{\infty} E_{\Delta_{n}}\right] U_{-t}=\bigvee_{n=0}^{\infty}\left[U_{t} E_{\Delta_{n}} U_{-t}\right] \\
& =\bigvee_{n=0}^{\infty} E_{\Delta_{n}+t}=E
\end{aligned}
$$

where the third equality follows from time-translation covariance. 
In order to prove the next result, we will need to invoke the following lemma from Borchers (1967).

Lemma (Borchers). Let $U_{t}=e^{i t H}$, where $H$ is a self-adjoint operator with spectrum bounded from below. Let $E$ and $F$ be projection operators such that $E F=0$. If there is an $\epsilon>0$ such that

$$
\left[E, U_{t} F U_{-t}\right]=0, \quad 0 \leq t<\epsilon,
$$

then $E U_{t} F U_{-t}=0$ for all $t \in \mathbb{R}$.

Lemma 4. Let $U_{t}=e^{i t H}$, where $H$ is a self-adjoint operator with spectrum bounded from below. Let $\left\{E_{n}: n=0,1,2, \ldots\right\}$ be a family of projection operators such that $E_{0} E_{n}=0$ for all $n \geq 1$, and let $E=\bigvee_{n=0}^{\infty} E_{n}$. If $U_{t} E U_{-t}=E$ for all $t \in \mathbb{R}$, and if for each $n \geq 1$ there is an $\epsilon_{n}>0$ such that

$$
\left[E_{0}, U_{t} E_{n} U_{-t}\right]=0, \quad 0 \leq t<\epsilon_{n},
$$

then $U_{t} E_{0} U_{-t}=E_{0}$ for all $t \in \mathbb{R}$.

Proof. If $E_{0}=0$ then the conclusion obviously holds. Suppose then that $E_{0} \neq 0$, and let $\psi$ be a unit vector in the range of $E_{0}$. Fix $n \geq 1$. Using (12) and Borchers' lemma, it follows that $E_{0} U_{t} E_{n} U_{-t}=0$ for all $t \in \mathbb{R}$. Then,

$$
\begin{aligned}
\left\|E_{n} U_{-t} \psi\right\|^{2} & =\left\langle U_{-t} \psi, E_{n} U_{-t} \psi\right\rangle=\left\langle\psi, U_{t} E_{n} U_{-t} \psi\right\rangle \\
& =\left\langle E_{0} \psi, U_{t} E_{n} U_{-t} \psi\right\rangle=\left\langle\psi, E_{0} U_{t} E_{n} U_{-t} \psi\right\rangle=0,
\end{aligned}
$$

for all $t \in \mathbb{R}$. Thus, $E_{n} U_{-t} \psi=0$ for all $n \geq 1$, and consequently, [ $\left.\bigvee_{n>1} E_{n}\right] U_{-t} \psi=$ 0 . Since $E_{0}=E-\left[\bigvee_{n \geq 1} E_{n}\right]$, and since (by assumption) $E U_{-t}=\bar{U}_{-t} E$, it follows that

$$
E_{0} U_{-t} \psi=E U_{-t} \psi=U_{-t} E \psi=U_{-t} \psi,
$$

for all $t \in \mathbb{R}$.

Theorem 1. Suppose that the localization system $\left(\mathcal{H}, \Delta \mapsto E_{\Delta}, t \mapsto U_{t}\right)$ satisfies localizability, probability conservation, time-translation covariance, energy bounded below, and microcausality. Then $U_{t} E_{\Delta} U_{-t}=E_{\Delta}$ for all $\Delta$ and all $t \in \mathbb{R}$.

Proof. Let $\Delta$ be an open subset of $\Sigma$. If $\Delta=\Sigma$ then probability conservation and time-translation covariance entail that $E_{\Delta}=E_{\Delta+t}=U_{t} E_{\Delta} U_{-t}$ for all $t \in \mathbb{R}$. If $\Delta \neq \Sigma$ then, since $\Sigma$ is a manifold, there is a covering $\left\{\Delta_{n}: n \in \mathbb{N}\right\}$ of $\Sigma \backslash \Delta$ such that the distance between $\Delta_{n}$ and $\Delta$ is nonzero for all $n$. Let $E_{0}=E_{\Delta}$, and 
let $E_{n}=E_{\Delta_{n}}$ for $n \geq 1$. Then 1 entails that $E_{0} E_{n}=0$ when $n \geq 1$. If we let $E=\bigvee_{n=0}^{\infty} E_{n}$ then probability conservation entails that $U_{t} E U_{-t}=E$ for all $t \in \mathbb{R}$ (see Lemma B). By time-translation covariance and microcausality, for each $n \geq 1$ there is an $\epsilon_{n}>0$ such that

$$
\left[E_{0}, U_{t} E_{n} U_{-t}\right]=0, \quad 0 \leq t<\epsilon_{n} .
$$

Since the energy is bounded from below, Lemma 4 entails that $U_{t} E_{0} U_{-t}=E_{0}$ for all $t \in \mathbb{R}$. That is, $U_{t} E_{\Delta} U_{-t}=E_{\Delta}$ for all $t \in \mathbb{R}$.

\section{B Appendix}

Theorem 2. Suppose that the unsharp localization system $\left(\mathcal{H}, \Delta \mapsto A_{\Delta}, \mathbf{a} \mapsto\right.$ $U(\mathbf{a}))$ satisfies additivity, translation covariance, energy bounded below, microcausality, and no absolute velocity. Then $A_{\Delta}=0$ for all $\Delta$.

Proof. We prove by induction that $\left\|A_{\Delta}\right\| \leq(2 / 3)^{m}$, for each $m \in \mathbb{N}$, and for each bounded $\Delta$. For this, let $F_{\Delta}$ denote the spectral measure for $A_{\Delta}$.

(Base case: $m=1)$ Let $E_{\Delta}=F_{\Delta}(2 / 3,1)$. We verify that $\left(\mathcal{H}, \Delta \mapsto E_{\Delta}, \mathbf{a} \mapsto\right.$ $U(\mathbf{a}))$ satisfies the conditions of Malament's theorem. Clearly, no absolute velocity and energy bounded below hold. Moreover, since unitary transformations preserve spectral decompositions, translation covariance holds; and since spectral projections of compatible operators are also compatible, microcausality holds. To see that localizability holds, let $\Delta$ and $\Delta^{\prime}$ be disjoint bounded subsets of a single hyperplane. Then microcausality entails that $\left[A_{\Delta}, A_{\Delta^{\prime}}\right]=0$, and therefore $E_{\Delta} E_{\Delta^{\prime}}$ is a projection operator. Suppose for reductio ad absurdum that $\psi$ is a unit vector in the range of $E_{\Delta} E_{\Delta^{\prime}}$. By additivity, $A_{\Delta \cup \Delta^{\prime}}=A_{\Delta}+A_{\Delta^{\prime}}$, and we therefore obtain the contradiction:

$$
1 \geq\left\langle\psi, A_{\Delta \cup \Delta^{\prime}} \psi\right\rangle=\left\langle\psi, A_{\Delta} \psi\right\rangle+\left\langle\psi, A_{\Delta^{\prime}} \psi\right\rangle \geq 2 / 3+2 / 3
$$

Thus, $E_{\Delta} E_{\Delta^{\prime}}=0$, and Malament's theorem entails that $E_{\Delta}=0$ for all $\Delta$. Therefore, $A_{\Delta}=A_{\Delta} F_{\Delta}(0,2 / 3)$ has spectrum lying in $[0,2 / 3]$, and $\left\|A_{\Delta}\right\| \leq 2 / 3$ for all bounded $\Delta$.

(Inductive step) Suppose that $\left\|A_{\Delta}\right\| \leq(2 / 3)^{m-1}$ for all bounded $\Delta$. Let $E_{\Delta}=F_{\Delta}\left((2 / 3)^{m},(2 / 3)^{m-1}\right)$. In order to see that Malament's theorem applies to $\left(\mathcal{H}, \Delta \mapsto E_{\Delta}, \mathbf{a} \mapsto U(\mathbf{a})\right)$, we need only check that localizability holds. For this, suppose that $\Delta$ and $\Delta^{\prime}$ are disjoint subsets of a single hyperplane. By microcausality, $\left[A_{\Delta}, A_{\Delta^{\prime}}\right]=0$, and therefore $E_{\Delta} E_{\Delta^{\prime}}$ is a projection operator. Suppose

for reductio ad absurdum that $\psi$ is a unit vector in the range of $E_{\Delta} E_{\Delta^{\prime}}$. Since 
$\Delta \cup \Delta^{\prime}$ is bounded, the induction hypothesis entails that $\left\|A_{\Delta \cup \Delta^{\prime}}\right\| \leq(2 / 3)^{m-1}$. By additivity, $A_{\Delta \cup \Delta^{\prime}}=A_{\Delta}+A_{\Delta^{\prime}}$, and therefore we obtain the contradiction:

$$
(2 / 3)^{m-1} \geq\left\langle\psi, A_{\Delta \cup \Delta^{\prime}} \psi\right\rangle=\left\langle\psi, A_{\Delta} \psi\right\rangle+\left\langle\psi, A_{\Delta^{\prime}} \psi\right\rangle \geq(2 / 3)^{m}+(2 / 3)^{m} .
$$

Thus, $E_{\Delta} E_{\Delta^{\prime}}=0$, and Malament's theorem entails that $E_{\Delta}=0$ for all $\Delta$. Therefore, $\left\|A_{\Delta}\right\| \leq(2 / 3)^{m}$ for all bounded $\Delta$.

\section{Appendix}

Theorem 3. Suppose that the system $\left(\mathcal{H}, \Delta \mapsto N_{\Delta}, \mathbf{a} \mapsto U(\mathbf{a})\right)$ of local number operators satisfies additivity, translation covariance, energy bounded below, number conservation, microcausality, and no absolute velocity. Then, $N_{\Delta}=0$ for all bounded $\Delta$.

Proof. Let $N$ be the unique total number operator obtained from taking the sum $\sum_{n} N_{\Delta_{n}}$ where $\left\{\Delta_{n}: n \in \mathbb{N}\right\}$ is a disjoint covering of $\Sigma$. Note that for any $\Delta \subseteq \Sigma$, we can choose a covering containing $\Delta$, and hence, $N=N_{\Delta}+A$, where $A$ is a positive operator. By microcausality, $\left[N_{\Delta}, A\right]=0$, and therefore $\left[N_{\Delta}, N\right]=\left[N_{\Delta}, N_{\Delta}+A\right]=0$. Furthermore, for any vector $\psi$ in the domain of $N,\left\langle\psi, N_{\Delta} \psi\right\rangle \leq\langle\psi, N \psi\rangle$.

Let $E$ be the spectral measure for $N$, and let $E_{n}=E(0, n)$. Then, $N E_{n}$ is a bounded operator with norm at most $n$. Since $\left[E_{n}, N_{\Delta}\right]=0$, it follows that

$$
\left\langle\psi, N_{\Delta} E_{n} \psi\right\rangle=\left\langle E_{n} \psi, N_{\Delta} E_{n} \psi\right\rangle \leq\left\langle E_{n} \psi, N E_{n} \psi\right\rangle \leq n,
$$

for any unit vector $\psi$. Thus, $\left\|N_{\Delta} E_{n}\right\| \leq n$. Since $\bigcup_{n=1}^{\infty} E_{n}(\mathcal{H})$ is dense in $\mathcal{H}$, and since $E_{n}(\mathcal{H})$ is in the domain of $N_{\Delta}$ (for all $n$ ), it follows that if $N_{\Delta} E_{n}=0$, for all $n$, then $N_{\Delta}=0$. We now concentrate on proving the antecedent.

For each $\Delta$, let $A_{\Delta}=(1 / n) N_{\Delta} E_{n}$. We show that the structure $(\mathcal{H}, \Delta \mapsto$ $\left.A_{\Delta}, \mathbf{a} \mapsto U(\mathbf{a})\right)$ satisfies the conditions of Theorem 2 . Clearly, energy bounded below and no absolute velocity hold. It is also straightforward to verify that additivity and microcausality hold. To check translation covariance, we compute:

$$
\begin{aligned}
U(\mathbf{a}) A_{\Delta} U(\mathbf{a})^{*} & =U(\mathbf{a}) N_{\Delta} E_{n} U(\mathbf{a})^{*}=U(\mathbf{a}) N_{\Delta} U(\mathbf{a})^{*} U(\mathbf{a}) E_{n} U(\mathbf{a})^{*} \\
& =U(\mathbf{a}) N_{\Delta} U(\mathbf{a})^{*} E_{n}=N_{\Delta+\mathbf{a}} E_{n}=A_{\Delta+\mathbf{a}} .
\end{aligned}
$$

The third equality follows from number conservation, and the fourth equality follows from translation covariance. Thus, $N_{\Delta} E_{n}=A_{\Delta}=0$ for all $\Delta$. Since this holds for all $n \in \mathbb{N}, N_{\Delta}=0$ for all $\Delta$. 


\section{REFERENCES}

Barrett, Jeffrey A. (2000), "On the nature of measurement records in relativistic quantum field theory", manuscript.

Bell, John S. (1987), Speakable and Unspeakable in Quantum Mechanics. New York: Cambridge University Press.

Borchers, H.-J. (1967), "A remark on a theorem of B. Misra”, Communications in Mathematical Physics 4: 315-323.

Buchholz, Detlev (2000), "Algebraic quantum field theory: A status report", math-ph/0011044.

Busch, Paul (1999), "Unsharp localization and causality in relativistic quantum theory", Journal of Physics A 32: 6535-6546.

Chaiken, Jan M. (1968), "Number operators for representations of the canonical commutation relations", Communications in Mathematical Physics 8: 164184.

Dickson, W. Michael (1998), Quantum Chance and Nonlocality. New York: Cambridge University Press.

Earman, John (1986), A Primer on Determinism. Boston: D. Reidel.

Fleming, Gordon, and Jeremy Butterfield (1999), "Strange positions", in J. Butterfield and C. Pagonis (eds.), From Physics to Philosophy. NY: Cambridge University Press, 108-165.

Haag, Rudolf (1992), Local Quantum Physics. New York: Springer.

Halvorson, Hans (2001), "Reeh-Schlieder defeats Newton-Wigner: On alternative localization schemes in relativistic quantum field theory", Philosophy of Science, forthcoming.

Hegerfeldt, Gerhard C. (1998a), "Causality, particle localization and positivity of the energy", in A. Böhm, et al. (eds.), Irreversibility and Causality. New York: Springer, 238-245.

Hegerfeldt, Gerhard C. (1998b), "Instantaneous spreading and Einstein causality in quantum theory", Annalen der Physik 7: 716-725.

Holland, Peter R. (1993), The Quantum Theory of Motion. New York: Cambridge University Press.

Malament, David (1996), "In defense of dogma: Why there cannot be a relativistic quantum mechanics of (localizable) particles", in Rob Clifton (ed.), Perspectives on Quantum Reality. Dordrecht: Kluwer, 1-10.

Maudlin, Tim (1994), Quantum Non-Locality and Relativity. Cambridge: Blackwell.

Redhead, Michael (1995), "The vacuum in relativistic quantum field theory", in David Hull, Micky Forbes, and Richard M. Burian (eds.), PSA 1994, v. 2. East Lansing, MI: Philosophy of Science Association, 77-87. 
Requardt, Manfred (1982), "Spectrum condition, analyticity, Reeh-Schlieder and cluster properties in non-relativistic Galilei-invariant quantum theory", Journal of Physics A 15: 3715-3723.

Schlieder, S. (1971), "Zum kausalen Verhalten eines relativistischen quantenmechanischen System", in S.P. Dürr (ed.), Quanten und Felder. Braunschweig: Vieweg, 145-160.

Segal, Irving E. (1964), "Quantum fields and analysis in the solution manifolds of differential equations", in William T. Martin and Irving E. Segal, (eds.), Proceedings of a Conference on the Theory and Applications of Analysis in Function Space. Cambridge: MIT Press, 129-153.

Streater, Raymond F. and Arthur S. Wightman (2000), PCT, Spin and Statistics, and All That. Princeton: Princeton University Press.

Thaller, Bernd (1992), The Dirac Equation. New York: Springer.

Ticiatti, Robin (1999), Quantum Field Theory for Mathematicians. New York: Cambridge University Press. 